\title{
Segmenting Sentences into Linky Strings Using D-bigram Statistics
}

\author{
Shiho Nobesawa \\ Junya Tsutsumi, Sun Da Jiang, Tomohisa Sano, Kengo Sato \\ Masakazu Nakanishi
}

\author{
Nakanishi Laboratory, Keio University \\ 3-14-1 Hiyoshi, Kohoku-ku \\ Yokohama 223 Japan \\ shiho@nak.math.keio.ac.jp
}

\begin{abstract}
It is obvious that segmentation takes an important role in natural language processing(NLP), especially for the languages whose sentences are not easily separated into morphemes. In this study we propose a method of segmenting a sentence. The systern described in this paper does not use any grammatical information or knowledge in processing. Instead, it uses statistical information drawn from non-tagged corpus of the target language. Most of the segmenting systems are to pick out conventional morphemes which is defined for human use. However, we still do not know whether those conventional morphemes are good units for computational processing.

In this paper we explain our system's algorithm and its experimental results on Japanese, though this system is not designed for a particular language.
\end{abstract}

\section{Characteristics of Japanese Text}

\subsection{Letters in Japanese}

Japanese text is composed of four kinds of characters - kanji, hiragana, katakana, and others such as alphabetic characters and numeral characters. Hiragana is used for Japanese words, inflections and function words, while katakana is used for words from foreign languages and for other special purposes.

Table 1 shows examples of rates of those four characters in texts (Teller and Batchelder, 1994). The bus. corpus consists of a set of newspaper articles on business ventures from Yomiuri. The ed. corpus contains a series of editorial columns from Asahi Shinbun.

Table 1: Character Rates in Japanese Text

$\begin{array}{lrr} & \text { bus. } & \underline{\text { ed. }} \\ \text { size(K chars) } & 42 & 275 \\ \text { \% hiragana } & 30.2 & 58.0 \\ \text { \% kanji } & 47.5 & 34.6 \\ \text { \% katakara } & 19.3 & 4.8 \\ \text { \% num/alph } & 2.9 & 2.6\end{array}$

\subsection{Morphemes in Japanese}

Segmenting a Japanese text is a difficult task. $\Lambda$ phrase “勉㤝していました (was studying)" can be a single lexical unit or can be separated into as many as six elements (Teller and Batchelder, 1994):

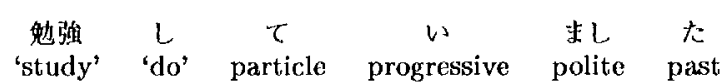

Acquiring "morphemes" from Japanese text is not a simple task because of this flexibility.

\section{Linky Strings}

This paper is on dividing non-separated language sentences into meaningful strings of letters without using any grammar or linguistic knowledge. Instead, this system uses the statistical information between letters to select the best ways to segment sentences in non-separated languages.

It is not very hard to divide a sentence using a certain dictionary for that. The problem is that a 'certain dictionary' is not easily obtainable. There never is a perfect dictionary which holds all the words that exist in the language. Moreover, building a dictionary is very hard work, since there are no perfect autornatic dictionary-making systems. 
Ilowever, machine-readable dictionaries are needed anyway. For this reason, we propose a new method for picking out meaningful strings. Our purpose is not to segment a sentence into conventional morphenes. We introduce a concept for a type of language unit for machine use. We named the unit a 'linky string'. A linky string is a series of letters extracted from a corpus using statistical information only. It is a series of letters which share a strong statistical relationship.

\section{Linking SCORE}

\subsection{Linking Score}

To pick out linky strings, wo need to find highly connectable letters in a sentence. We introduce the linking score, which shows the lirkability between two neighbor letters in a scntence. This score is estimated using d-bigram statistics.

\subsection{D-bigram}

The idea of bigrams and trigrams is often used in studies on NI,P. N-gram is the information of the association between $n$ certain events. In this study we use the d-bigram data (T'sutsumi et al., 1993), which is a kind of bigram data with the concept of distance between events (Figure 1). D-bigram is equal to bigram when $d=1$, thus d-bigram data includes the conventional bigram relation.

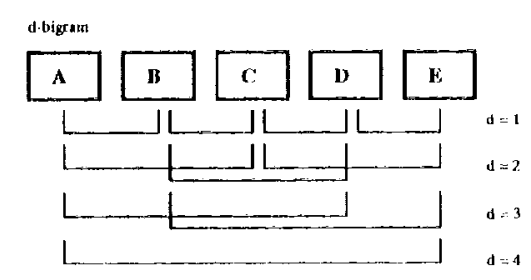

Figure 1: D-bigram

\subsection{Calculation}

\section{Mutual Information with Distance}

Hxpression (1) is for calculating inutual infornation between two events(Nobesawa et al., 1994):

$$
M I\left(a_{i}, b_{j}, d\right)=\log _{2} \frac{P\left(a_{i}, b_{j}, d\right)}{P\left(a_{i}\right) P\left(b_{j}\right)}
$$

$$
\begin{array}{lll}
a_{i} & : & \text { a letter } \\
P\left(a_{i}\right) & : & \text { the possibility the letter } a_{i} \text { appears } \\
P\left(a_{i}, b_{j}, d\right) & : & \text { the possibility } a_{i} \text { and } b_{j} \text { appear together } \\
& & \text { with the distance } d \text { in a sentence }
\end{array}
$$

The parameter $d$ shows the distance between two events. In Figure 2, the distance between "a" and "pen" is 1, and the distance between "is" and "pen" is 2 as well. Since the event order has a meaning, in this case the distance between "pen" and " $\mathrm{a}$ " is defined as -1 .

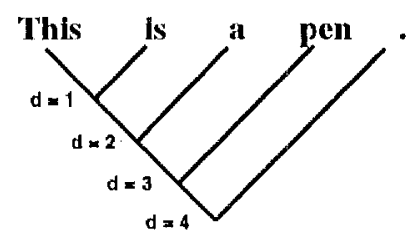

Figure 2: D-bigram Example

As the value of $M l$ gets bigger, the stronger is the association between the two events.

\section{Linking Score}

Lxpression (2) is for calculating the linking score between two letters in a sentence ${ }^{1}$.

$$
\begin{aligned}
& U K[i]=\sum_{d=1}^{d_{\max }} \sum_{j=i-(d-1)}^{i} M I\left(w_{j}, w_{j+d}, d\right) \cdot g(d) \\
& d_{\max }: \text { max distance used } \\
& u_{i} \quad \text { : the } i \text {-th letter in the sentence } w \\
& g(d) \quad \text { : a certain weight for } M \\
& \text { concerning distance between letters }
\end{aligned}
$$

'The information between two remote words has less meaning in a sentence when it comes to the semantic analysis(Church and Hanks, 1989). According to the idea we put $g(d)$ in the expression so that nearer pair can be more effoctive in calculating the score of the sentence.

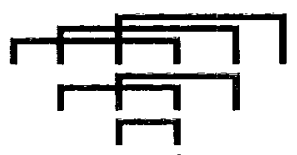

A B C (D) F G H

Figure 3: Calculation of I Jinking Score

A pair of far-away letters do not have strong relation between each other, neither syntactically nor semantically. For this reason we use $d_{\max }$, and in this paper we set the $d_{\max }$ value ${ }^{2}$ to 5 and 1 . When the $d_{\max }$ is 1 , the $M I$ used in calculation is only bigram data.

\footnotetext{
"Wo made a Japanese word "你" for the word "linky". We used it's pronunciation "UK [ju:kei]" in the expression.

${ }^{2}$ We had experiments for finding a good value for $d_{\max }$.
} 


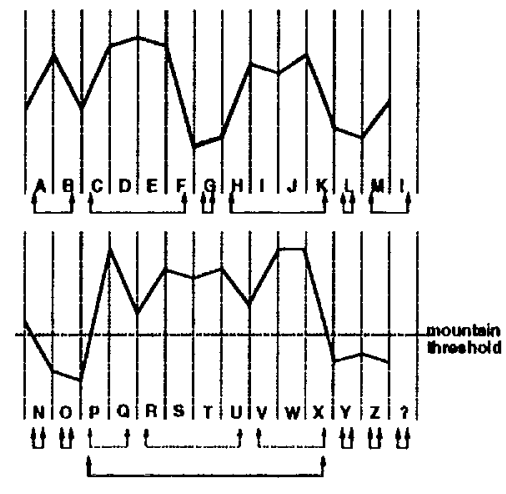

Figure 5: The Score Graph

\section{The System LSS}

\subsection{Overview}

This system is called LSS, a "linky string segmentor". This system takes a corpus made of nonseparated sentences as its input and segments it into linky strings using d-bigram statistics.

Figure 4 shows the flow of ISS's processing.

Input sentences to segment.
$\Downarrow$
Calculate the linking score of
each pair of neighboring letters.
$\Downarrow$
Check the score graph
to see where to segment.
$\Downarrow$
pick out each linky string
found in the given corpus.

Figure 4: System Processing Flow

In this paper we used a fixed score for the starting score, so that ISS can decide whether the first letter should be a one-letter linky string.

\subsection{The Score Graph}

\section{What a Score Graph Is}

To segment a sentence into statistically-meaningful strings, we use the linking scores to locate boundaries between linking strings. A score graph has the letters in a sentence on the $\mathrm{x}$-axis and linking scores on the $y$-axis (Figure 5). We get one score graph for each sentence. Figure 5 shows two sentences (one above and one below), each of 14 letters (including an exclamation/question mark as the sentence terminator).
When the linking score between a pair of neighboring letters is high, we assume they are part of the same word. When it is low, we assume that the letters, though neighbors, are statistically independent of one another. In a score graph, a series of scores in the shape of mountain (ex.: A-B and C-F part in Figure 5) becomes a linky string, and a valley (ex.: between the letter $B$ and $C$ in Figure 5) is a spot to segment.

\section{Score-Graph Segmenting Algorithm}

The system LSS finds the valley-points in a sentence and segments the sentence there into strings.

Following is the algorithm to find the segmenting points in a sentence.

1. Do not segment in a mountain.

2. Segment at the valley point.

3. Cut before and after a one-lettered linky string.

\section{One-Lettered Linky String}

A one-lettered linky string needs to (a) place at the valley point, and (b) look flat ${ }^{3}$ in the score graph. In Figure 5, one-lettered linky strings are $\mathrm{G}, \mathrm{L}, \mathrm{N}^{4}, \mathrm{O}, \mathrm{Y}, \mathrm{Z}$ and ?.

\section{Mountain Threshold}

A linky string takes a mountain shape because of high linking scores. Note that a linky string is not equal to a morpheme in human-handmade grammars. When a certain pair of morphemes occurs in a corpus very often, the system recognizes the pair's high linking score and puts them together into one linky string. For example, “ブッシコ大 統領 (President Bush)" is often treated as a linky string, since “ブッシュ(Bush)" and “大統領 (president)" appear next to each other very frequently.

The mountains of letters are not always simple hat-shaped; most of time they have other smaller mountains in them. This means that there can be shorter strings in one linky string. In one linky string “ブッシュ大統領 (President Bush)", there must be two smaller mountains, just like $\mathrm{H}-\mathrm{I}$ and $\mathrm{J}-\mathrm{K}$ in the mountain $\mathrm{H}-\mathrm{K}$ in Figure 5. To control the size of linky strings we introduce a mountain threshold, which is shown in the sentence below in Figure 5. When the score of a valley point is higher than the mountain threshold, the system judges the point is not a segmenting spot. In this paper the mountain threshold value is 5.0 .

\footnotetext{
${ }^{3}$ We use a constant value as a threshold.

${ }^{4} \mathrm{~N}$ is a special one-lettered linky string which places at the beginning of a sentence.
} 


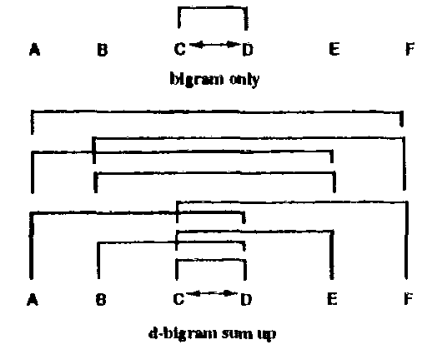

Figure 6: The Difference between D-bigram and Bigram

\subsection{Corpus}

ISS accepts all the non-separated sentences with little preparation. All we need is a set of certain amount of the target-language corpus for training.

In this paper we show the experimental results on Japanese. The corpus prepared for this paper is of Asahi Shinbun Newspaper.

\section{Results}

\subsection{Experimental Results}

\section{Experiment Condition}

ISS takes a set of non-separated sentences as its input and segments them into linky strings. For the test corpus we chose sentences at random from the training corpus.

Table 2: Training Corpus Condition

\begin{tabular}{|c|c|}
\hline $\begin{array}{l}\text { language: } \\
\text { form: }\end{array}$ & $\begin{array}{l}\text { Japanese } \\
\text { non-separated }\end{array}$ \\
\hline $\begin{array}{r}\text { corpus: } \\
\text { \# of sentences }\end{array}$ & Asahi Shinbun Newspaper \\
\hline $\begin{array}{r}\text { for training corpus: } \\
\# \text { of sentences }\end{array}$ & 7,502 \\
\hline for test corpus: & 302 \\
\hline
\end{tabular}

'To see the efficacy of d-bigram, we compare the experimental results of two data: d-bigram data and bigram data.

\section{Experimental Results}

As shown in Table 3, with d-bigram information only $7.39 \%$ of the segment spots are over-segmented.

Table 3 shows that a sentence gets separated
Table 3: Output

\begin{tabular}{|l|r|r|}
\hline & $\begin{array}{r}\text { d-bigram } \\
d_{\text {max }}=5\end{array}$ & bigram \\
\hline \hline $\begin{array}{l}\text { \# of input sentences } \\
\text { \# of linky strings } \\
\text { \# of linky strings per } \\
\text { sentence }\end{array}$ & 302 & 302 \\
\hline $\begin{array}{l}\text { \# of over-segmented spots } \\
\text { over-segmented spots }\end{array}$ & 20.35 & 23,098 \\
\hline
\end{tabular}

into 20-25 linky strings on average ${ }^{5}$. And in one sentence there are only one or two spots on average which break a morpheme into meaningless strings. With no linguistic knowledge, this can be said to be quite a good result.

It is hard to check whether an extracted linky string is a right one, however, it is not that difficult to find over-segmented strings, for a linky string needs to hold the meaning. We check those oversegmented linky strings according to a dictionary, Iwanami Kokugo Jiten.

Table 4 shows the numbers of over-segmented spots. The figure is the number of over-segmented spots, not the number of morphemes over-segmented ${ }^{6}$. In Table $4 \mathrm{~A}$ and $\mathrm{B}$ are neighboring letters in a sentence which are forced to separate. The row "kanji hiragana" stands for over-segmented spots between a kanji letter and a hiragana letter.

Table 4: Over-Segmented Morphemes by Character Types and Segmentation Methods

\begin{tabular}{|c|c|c|c|}
\hline A & B & $\begin{array}{l}\text { d-bigram } \\
d_{\max }=5\end{array}$ & bigram \\
\hline kanji & kanji & 59 & 65 \\
\hline kanji & hiragana & 29 & 43 \\
\hline hiragana & kaniji & 18 & 22 \\
\hline hiragana & hiragana & 333 & 507 \\
\hline katakana & katakana & 15 & 52 \\
\hline & total & 454 & 689 \\
\hline
\end{tabular}

The ratio of over-segmented morphemes for each part of speech is shown in Table 5 . ' $\mathrm{K}$ ' stands for kanji, ' $h$ ' is for hiragana and ' $k$ ' is for katakana.

There was no missegmentation between katakana and other character types. There also was not any

\footnotetext{
${ }^{5}$ The range of numbers of linky strings found in a sentence is 5-60 with d-bigram and 6-66 with bigram.

${ }^{6}$ Thus a morpheme gets counted twice when it is divided into three strings.
} 
Table 5: Over-Segmented Morphemes in Output with D-bigram

\begin{tabular}{|c|rrrrr|r|}
\hline $\begin{array}{c}\text { A } \\
\text { B }\end{array}$ & $\begin{array}{r}\mathrm{K} \\
\mathrm{K}\end{array}$ & $\begin{array}{r}\mathrm{K} \\
\mathrm{h}\end{array}$ & $\begin{array}{c}\mathrm{h} \\
\mathrm{K}\end{array}$ & $\begin{array}{c}\mathrm{h} \\
\mathrm{h}\end{array}$ & $\begin{array}{r}\mathrm{k} \\
\mathrm{k}\end{array}$ & total \\
\hline noun & 49 & 19 & 6 & 49 & 6 & 129 \\
proper noun & 5 & & & & 8 & 13 \\
pronoun & & & & 16 & & 16 \\
\hline verb & $\mathrm{I}$ & 3 & 12 & 84 & & 100 \\
aux. verb & & & & 60 & & 60 \\
adjective & & 4 & & 12 & & 16 \\
adj. verb & 4 & & & 13 & & 17 \\
\hline adverb & & 1 & & 53 & 1 & 55 \\
rentai-shi & & & & 11 & & 11 \\
conjunction & & & & 7 & 7 & 7 \\
funcion word & & & & 15 & & 15 \\
suffix & & 1 & & 4 & & 5 \\
compound word & & 1 & & 15 & 16 \\
\hline \hline
\end{tabular}

missegmentation concerning alphabets, numeral characters and other symbols.

\subsection{A Linky String}

\section{Characteristics of Linky Strings}

Linky strings in Japanese are not equal to conventional morphemes in Japanese. As discussed in section 1.2 , it is not easy to decide an absolutely correct segmenting spot in a Japanese sentence. That is one of the reasons that we decided to extract linky strings, instead of conventional morphemes. However, if those linky strings do not keep the meanings, it is useless.

The result shows the linking score works well enough not to segment senteces too much (Table 3). That is, we succecded in extracting meaningful strings using only statistical information. Figure 7 shows some examples of extracted linky strings.

$\begin{array}{ccc}\text { 銀行 } & \text { bank(s) } & \text { meaningful } \\ \text { に移行 } & \text { move/shift to } & \text { meaningful } \\ \text { の)行動 } & \text { action of } & \text { meaningful } \\ \text { を行った } & \text { did } & \text { meaningful } \\ \text { (?) 行(?) } & & \text { over-segmented } \\ \text { (年)中行事 } & & \text { over-segmented }\end{array}$

Figure 7: Examples of Linky Strings (1)

Sometimes LSS extracts strings that look too long (Figure 8). This is not a bad result, though. When a linky string contains several morphemes in it, it is something like picking out idioms. A linky string with several morphernes may be a compound word, or an idiom, or a fixed locution.

$$
\begin{aligned}
& \text { 手を貸小 } \\
& \text { ロンドン・サミット }
\end{aligned}
$$

Figure 8: Examples of Linky Strings (2)

\section{The Concept of the Linky Strings}

Grammar-based NLP systems generally specify a target language. On the other hand statisticallybased approachs do not need rules or knowledge. This makes a statistically-based approach suitable to multilingual processing.

ISS is not only for Japanese. With a corpus of non-separated sentences of any language, ISS can perform the same kind of segmentation.

To deal with natural languages most systems use conventional morphemes or words as their processing units. That is, most systems need to recognize morphemes or words in sentences, and they need to make up a fairly-good morphological analysis before the main processing. We have been working for processing natural languages in linguistic ways, though we do not know whether it is a right way in computational linguistics. A linky string is extracted only with statistical information, using no grammars nor linguistic knowledge. The system does not need to behave like a native speaker of the target language; all it has to do is check statistical information, which is what computers are good at. We expect that linky strings can be a key to solve problems of NLP.

\section{Compound Words}

The results show that the system has $7.39 \%$ incorrect segmentation. This result is based on a Japanese dictionary, and when a morpheme listed in the dictionary gets separated, we count it as over-segmented. However, a dictionary often holds compound words. That is, some number of the segmented spots which we have counted as "oversegmented" ones are not really over-segmented. From this point of view, the percentage of oversegmentation is actually even lower.

\section{Inflections}

Verbs, adjectives, adverbs and auxiliary verbs are inflected in Japanese. In the experimental result, 89.7\% (with d-bigram data) of over-segmented spots between kanji and hiragana occurs in inflective morphemes. We decided correct segmenting spots 
for inflective morphemes according to a Japanese dictionary. According to statistical information, segmenting method for inflective morphemes is different from grammatical one. So most of the oversegmented spots can be treated as correct segmenting spots according to statistical information.

\subsection{D-bigram Statistics}

According to Table 3 , it seems that using the bigram method the output is apt to be more segmented than with the d-bigram method.

This happens because bigram cannot pick out long strings. Bigram does not hold information between remote (actually more than one letter away) letters. That makes long strings of letters easily segmented. When ISS checks a three-letter morpheme $A B C$, with bigram data it can see the string only as $A-B$ and $B-C$. If those strings $A B$ and $B C$ do not appear often, the linking scores get low and ISS decides to segment between A$B$ and B-C. However, with d-bigram data ISS can get the information between $A$ and $C$ as well, that helps to recognize that $A, B$ and $C$ often come out together. This happens frequently between two katakana letters ('Table 4), because of the usage of katakana letters in Japanese.

This does not mean that with d-bigrarn method sentences are less likely to be segmented. As shown in Figure 9, the distribution is not so different between two methods. The $\mathrm{x}$-axis shows the numbers of linky strings in sentences and the $y$-axis shows the number of sentences with $x$ linky strings.

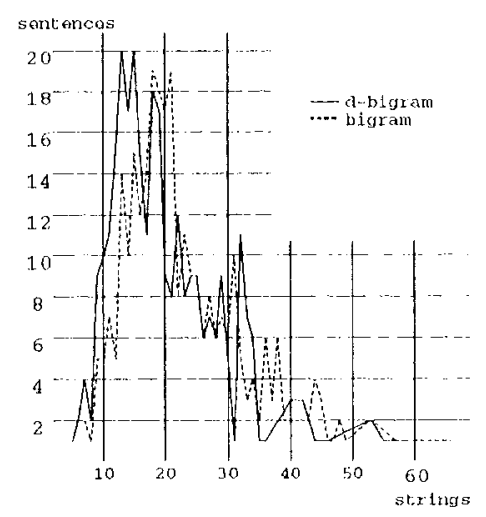

Figure 9: The Number of Strings in Output Sentences

According to Figure 9, the distributions of sentences are not so different between the method with d-bigram and the one with bigrarn.

\section{Conclusion}

This paper shows that this automatic segmenting system ISS is quite efficient for segmentation of non-separated language sentences. ISS does not use any grammatical information to divide input sentences into linky strings, that is, a new unit for NLP. According to the results of the experiments, LSS can segment almost all the sentences 'correctly', with strings kecping their meanings. This remarkable result of a statistic-based system ISS shows that d-bigran statistical information can be a key to meaningful-string extracting.

This result also shows that the concept of linky strings is an interesting concept for NLP. We expect that this linky string can be a unit for machinc translation systems or key word/phrase extraction systems, and other NLP systems.

\section{References}

[1] 'Tsutsumi, J., Nitta, T., Ono, K. and Nobesawa, S. A Multi-Lingual Translation System Based on A Statistical Model(written in Japanese). JSAI Technical report, SIG-PPAI-9302-2, pages 7-12, 1993.

[2] Nobesawa, S., Tsutsumi, J., Sun D. J., Sano, T., Sato K. and Nakanishi, M. Automatic Extraction of Linky Strings in Natural Languages (written in Japanese). 2nd Annual Meeting of the ANLP (NLPO6), pages 181-184, 1996.

[3] Nobesawa, S., Tsutsumi, J., Nitta, T., Ono, K., Sum, D. J. and Nakanishi, M. Segmeting a Japanese Sentence into Morphemes Using Statistical Information betwcen Words. Coling-94, pages 227-233, 1994.

[4] Teller V. and Batchelder E. O. A Probabilisitic Algorithm for Segmenting Non-Kanji Japanese Strings. $A A A I, 1994$.

[5] Brown, P., Cocke, J., Pietra, S. D., Pietra, V. D., Jelinek, F., Mercer, R. and Roossin, A. A Statistical Approach to Language Translation. Coling-88, pages 71--76, 1988.

[6] Church, K. and Hanks, P. Word Association Norms, Mutual Information, and Lexicography. In Proceedings of the 27th Annual Conference of the association of Computational Linguistics, 1989.

[7] Nishio, M., Iwabuchi, F. and Mizutani, S. Japanese-Japanese Dictionary The 3rd Edition. Iwanami Shoten, 1985. 